\title{
Origin of the refractive index modification of femtosecond laser processed doped phosphate glass
}

\author{
Andrés Ferrer, ${ }^{1}$ Daniel Jaque, ${ }^{2}$ Jan Siegel, ${ }^{1}$ Alexandro Ruiz de la Cruz, ${ }^{1}$ and Javier Solís ${ }^{1, a)}$ \\ ${ }^{1}$ Laser Processing Group, Instituto de Óptica, CSIC. Calle Serrano 121, 28006 Madrid, Spain \\ ${ }^{2}$ Fluorescence Imaging Group, Departamento de Física de Materiales, Facultad de Ciencias, \\ Universidad Autónoma de Madrid, 28049 Madrid, Spain
}

(Received 22 November 2010; accepted 3 March 2011; published online 6 May 2011)

\begin{abstract}
The origin of the local refractive index modification in femtosecond laser inscribed structures has been investigated with confocal microfluorescence imaging. We have identified the origin of both, positive and negative refractive index changes in a commercial Er- $\mathrm{Yb}$ codoped phosphate glass upon irradiation in the low repetition rate regime $(1 \mathrm{kHz})$. Consistent relations among the photoluminescence behavior of the dopants (erbium and ytterbium ions), the local sign of the density change and the local modification of the refractive index by means of characteristic emission features such as the intensity and the spectral peak position have been established. Blue or redshift in the photoluminescence emission have been observed and related to a local perturbation in the crystal field caused by a modification of the mean distance among the dopant ions, and thus of the local matrix density. These conclusions are additionally supported by the spatial distribution of photoluminescence emission intensities, which have been interpreted in terms of energy transfer mechanisms underlying the overall erbium-ytterbium emission process. (C) 2011 American Institute of Physics. [doi:10.1063/1.3572268]
\end{abstract}

\section{INTRODUCTION}

Micromachining of glasses using femtosecond (fs) laser direct writing has become a very popular technique over the last decade or so. Since it was first reported by Davis et al. ${ }^{1}$ in 1996, several photonic devices have been reported. These include devices based on waveguides such as amplifiers, lasers $^{2}$ or couplers, ${ }^{3}$ microfluidic devices based on microchannels, ${ }^{4}$ and lab on chip devices ${ }^{5}$ based on a combination of both waveguides and microchannels.

Femtosecond-laser direct writing is based on localized multiphoton absorption and subsequent avalanche ionization processes that occur in the focal region when focusing fs laser pulses inside a transparent dielectric. Under such conditions, the material undergoes local structural modifications that are typically accompanied by a change in refractive index in the focal region and its surroundings. A specific photonic device such as a waveguide (and all of its derivates), can be then written by translating the sample at constant velocity transversely to the beam propagation axis while irradiating the sample at constant repetition rate. Although this technique is quite extended, the mechanisms underlying the refractive index changes are still under investigation. While for a relatively simple material, such as fused silica, the mechanism is understood in the low repetition rate regime (densification and refractive index increase $^{6}$ ), for more technologically relevant materials such as phosphate glasses, the effort of several groups to determine these mechanisms is still ongoing.

Recent works propose two different mechanisms for the local increase of the refractive index in similar phosphate glasses depending on the repetition rate of the irradiation

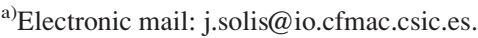

laser. In the high repetition rate regime $(\mathrm{MHz})$, Fletcher et al. ${ }^{7}$ have provided evidence for a local densification in the irradiated area. In the low-repetition rate regime $(\mathrm{kHz})$, Little et $a l .{ }^{8}$ have found strong indications for a structural modification in the glass network that locally increases the polarizability of the material without affecting the local density. It is worth noting though that the glass composition in both studies was different: Er-Yb codoped MM-2a60 (Ref. 7) and Yb doped QX (Ref. 8), both from Kigre Inc. Both works have been based on the interpretation of Raman spectra.

In the present paper we employ confocal microfluorescence imaging to study the modification of a similar material upon fs-laser excitation. This technique exploits the active ions embedded in the matrix as optical sensors inside the material. In particular, ions locally modify their emission properties such as peak wavelength, peak width and intensity, according to local changes of the matrix that occur upon laser irradiation. In crystals, this technique has proven extremely useful to understand and characterize modifications occurred upon femtosecond laser writing. ${ }^{9}$ In the present work, we demonstrate its suitability in an Er-Yb codoped phosphate glass, in particular in ultrafast laser inscribed waveguides. This technique allows us to infer the region where the photoluminescence of the $\mathrm{Er}$ and $\mathrm{Yb}$ ions and the energy transfer mechanisms have been modified, denoting in this way the presence of micro-structural modifications. The nature and extension of such micro-modifications is then discussed in terms of the fluorescence images.

\section{EXPERIMENTAL DETAILS}

In the present work, we have studied waveguides written inside a commercial doped phosphate glass (MM2, Kigre Inc., doped with 2.6 wt. $\%$ of Er and 2.9 wt $\%$ of $\mathrm{Yb}$ ). We 
TABLE I. Composition of the sample under study as measured by RBS.

\begin{tabular}{lccc}
\hline \hline Compound & Wt. $\%$ & Compound & Wt. \% \\
\hline $\mathbf{P}_{\mathbf{2}} \mathbf{O}_{\mathbf{5}}$ & $74.0 \pm 2.2$ & $\mathbf{M g O}$ & $<3$ \\
$\mathbf{A l}_{\mathbf{2}} \mathbf{O}_{\mathbf{3}}$ & $12.7 \pm 0.4$ & $\mathbf{N b}_{\mathbf{2}} \mathbf{O}_{\mathbf{5}}$ & $<3$ \\
$\mathbf{G e O}_{\mathbf{2}}$ & $4.16 \pm 0.12$ & $\mathbf{E r}_{\mathbf{2}} \mathbf{O}_{\mathbf{3}}$ & $<3$ \\
$\mathbf{L a}_{\mathbf{2}} \mathbf{O}_{\mathbf{3}}$ & $<3$ & $\mathbf{Y b}_{\mathbf{2}} \mathbf{O}_{\mathbf{3}}$ & $<3$ \\
\hline \hline
\end{tabular}

have performed Rutherford Back Scattering (RBS) measurements in order to determine the glass composition, which is shown in Table I.

For the microfluorescence measurements, an Argon laser at $488 \mathrm{~nm}$ has been used to excite the Erbium ions, and a laser diode at $980 \mathrm{~nm}$ has been used to excite the Ytterbium ions. These two pump wavelengths have been labeled in the absorption spectrum shown in Fig. 1. The pump lasers enter the excitation path of a confocal fluorescence microscope and are focused slightly below the sample surface by a $50 \mathrm{X}$ microscope objective ( 0.75 numerical apertures). The fluorescence/upconversion emission is then collected by the same microscope objective and, after passing some blocking filters and a confocal aperture, it is guided to enter an imaging spectrograph where a cooled charged coupled device (CCD) camera is attached. A typical emission spectrum is shown in Fig. 1 (bottom curve), where the peaks that we have studied in this work have been labeled. The sample is placed on a XY scanning stage (X and $\mathrm{Y}$ being the two dimensions perpendicular to the pump beam axis) in such a way that it is possible to scan the excitation spot over the cross sections of the waveguides. In particular, we have rastered the sample with a step size of $2 \mu \mathrm{m}$, the spot size being smaller than $1 \mu \mathrm{m}$. The resulting 4-D stack (X, Y, emission

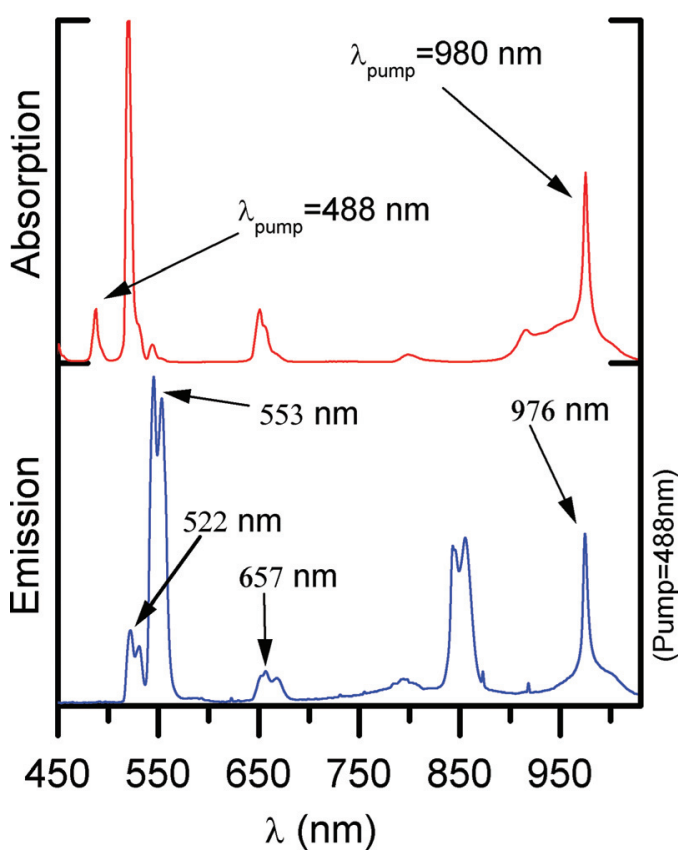

FIG. 1. (Color online) Top curve (red): normalized absorption spectrum of the Er-Yb codoped sample; the labeled peaks correspond to the pump wavelengths used in this study. Bottom curve (blue): normalized emission spectrum when pumping at $488 \mathrm{~nm}$; the peaks under study in the present paper have been labeled. wavelength, spectral intensity) can be then represented in multiple sample surface images that provide particular information such as the spatial variation of peak intensity, peak width and peak position.

We have performed microraman measurements (upon $488 \mathrm{~nm}$ excitation) in order to compare our results with those obtained by Fletcher et $a l^{7}$ and Little et $a .^{8}{ }^{8}$ Unfortunately, the $\mathrm{Er}^{3+}$ doping of our sample under $488 \mathrm{~nm}$ excitation (a resonant wavelength with the Erbium ions), produces a fluorescence band at $522 \mathrm{~nm}$ that masks the crucial Raman bands in such a way that no useful information could be extracted.

The waveguide writing setup consists of a commercial amplified femtosecond laser system $(120 \mathrm{fs}, 1 \mathrm{kHz}$ repetition rate) delivering pulses at a maximum energy of $1 \mathrm{~mJ}$. These pulses are stretched by detuning the compressor stage of the amplifier to a pulse duration of $260 \mathrm{fs}$. The circular laser beam $\left(1 / \mathrm{e}^{2}\right.$ diameter of $\left.7.4 \mathrm{~mm}\right)$ passes a $350 \mu \mathrm{m}$ wide slit in order to become strongly elliptical (so as to produce a circular cross section of the modified region), ${ }^{10}$ followed by a quarterwave plate to obtain circular polarization for two main reasons: on the one hand this polarization helps reducing the nonlinear propagation effects ${ }^{11,12}$ and, on the other it has been previously shown that it reduces propagation losses. ${ }^{13}$ The so-configured beam is focused by a microscope objective (numerical aperture of 0.26 , long-working distance) into the sample, to a depth of $1.56 \mathrm{~mm}$. The sample is translated at a constant speed of $100 \mu \mathrm{m} / \mathrm{s}$ in a direction perpendicular to the incident beam propagation axis, and parallel to the slit's long axis. Two different fs-laser written structures are studied. The first one (WG1) is a structure written with $4 \mu \mathrm{J}$ pulses, ensuring that nonlinear propagation effects are minimized as explained in Refs. 11 and 12. The second structure (WG2), is a waveguide that has been written with exactly the same parameters as WG1, however WG2 has been re-scanned over exactly the same region twenty times in order to improve the guiding properties as in Refs. 1, 14 and 15. After writing, the samples are polished at both end faces to bring the waveguides to the sample surface.

\section{RESULTS AND DISCUSSION}

In Fig. 2(a), a white-light transmission micrograph of the end facet of WG1 is shown. It features a dark ring with a gray region at the center, only slightly brighter than the background. In Fig. 2(b), the same end facet of WG1 is shown when attempting to couple $1550 \mathrm{~nm}$ light with a SMF28 fiber. This image shows that the structure is unable to support a guided mode at $1550 \mathrm{~nm}$, indicating that the laser induced refractive index change in the center is either positive and too low, or negative.

Figure 2(c) shows the spectral shift image of the Erbium photoluminescence (PL) peak with respect to the mean value of the unmodified area $(553 \mathrm{~nm})$. In this wavenumber representation, positive values indicate spectral shifts to shorter wavelengths, i.e., blueshifts. This figure has been treated digitally (smoothed) in order to reduce the noise and to reveal the weak but systematic blueshift appearing in the irradiated region. This blueshift is tentatively attributed to a reduction in the crystal field affecting Erbium ions. Thus, in a first 

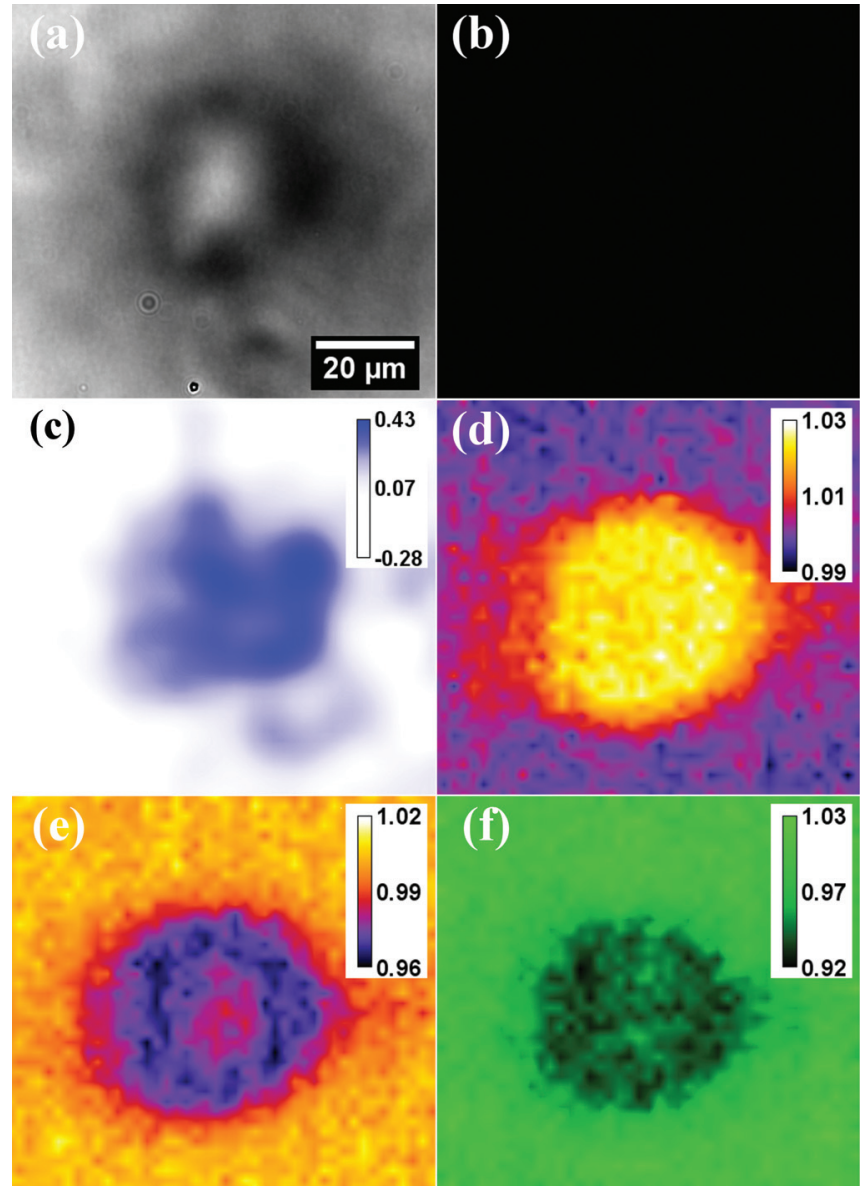

(f)

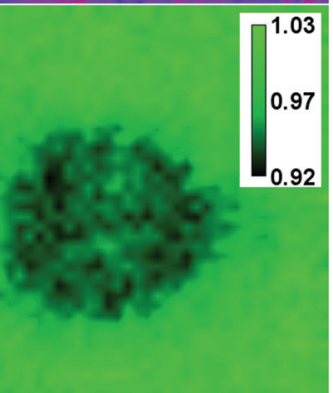

FIG. 2. (Color online) End facet of WG1 as seen with different characterization methods: (a) Transmission white light micrograph (b) Micrograph of WG1 when coupling light at $1550 \mathrm{~nm}$. (c) Spectral shift in $\mathrm{cm}^{-1}$ of the 553 $\mathrm{nm}$ Erbium emission line as obtained under $488 \mathrm{~nm}$ excitation. (d) Normalized $553 \mathrm{~nm}$ Erbium emission intensity as obtained under $488 \mathrm{~nm}$ excitation. (e) Normalized $976 \mathrm{~nm}$ Ytterbium emission intensity as obtained under 488 $\mathrm{nm}$ excitation. (f) Normalized $657 \mathrm{~nm}$ upconversion emission intensity of the Erbium ions under $980 \mathrm{~nm}$ excitation.

order approximation, it can be interpreted as an expansion of the matrix hosting the emitting ions, ${ }^{16}$ which leads to a local decrease in the refractive index. Even though this kind of measurement is more accurate for crystals, where emission peaks are narrower, and where even quantitative information has been obtained, ${ }^{9}$ it is still able to provide, in the case of glasses, qualitative features that can be then corroborated by further characterization, as shown next.

Figure 2(d) shows the $553 \mathrm{~nm}$ Erbium emission intensity image obtained after $488 \mathrm{~nm}$ optical excitation in the structure WG1. The intensity increases considerably in the fslaser irradiated area with respect to the bulk value. Assuming that there has been a local expansion of the matrix, as shown above, $\mathrm{Er} \rightarrow \mathrm{Yb}$ energy transfer is reduced by the larger donor-acceptor distance, yielding a reduced energy transfer probability. ${ }^{17}$ As a consequence, the PL emission of the Er at $553 \mathrm{~nm}$ is increased with respect to the bulk value. Following this same line of argument, one would expect a decrease in the Yb PL emission at $976 \mathrm{~nm}$ under $488 \mathrm{~nm}$ pump due to the reduction of the $\mathrm{Er} \rightarrow \mathrm{Yb}$ transfer. This effect can indeed be observed in Fig. 2(e), where the peak PL emission of the irradiated area is clearly below the bulk one.
On the other hand, the local density drop in the irradiated region not only affects the distance between $\mathrm{Er}$ and $\mathrm{Yb}$ ions, but also to the distance between the Er ions themselves. This fact, together with the reduction of the $\mathrm{Yb} \rightarrow$ Er energy transfer, should lead to a decrease in upconversion (UC) of the $\mathrm{Er}$ ions when pumped at $980 \mathrm{~nm}$. This is exactly the behavior observed in Fig. 2(f), where the irradiated region presents lower UC emission as compared to the nonirradiated one.

Summing up all the information obtained from the confocal microfluorescence images, we can conclude not only that the reason for WG1 to not to support a guided mode is a reduction of density and thus a decrease in the refractive index, but also that the density decrease of the matrix affects the distance among the embedded ions and their emission intensities. This fact is of particular interest when considering the optimum doping concentrations for fs-laser written active waveguides. $^{18}$

While WG1 is unable to support a guided mode at 1550 $\mathrm{nm}$ due to a refractive index depression, waveguiding can be achieved upon rescanning several times (twenty scans in this

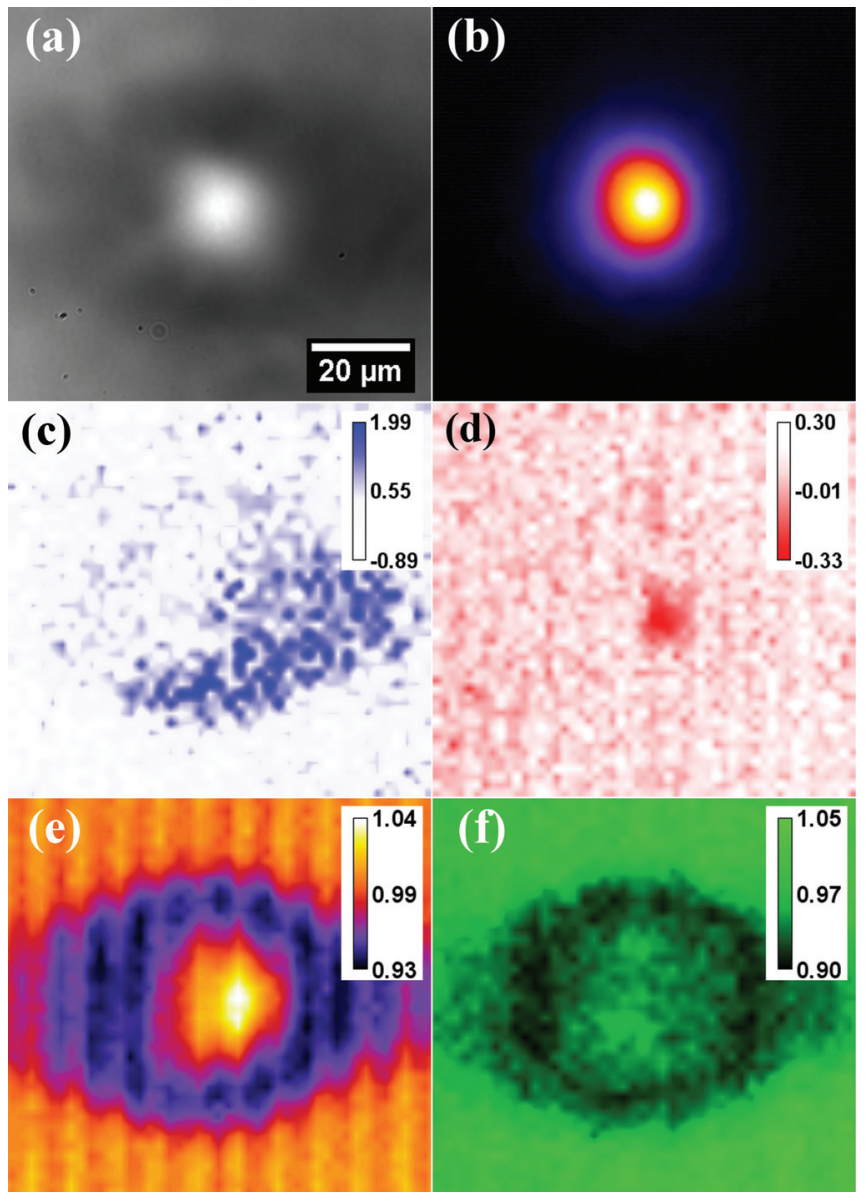

FIG. 3. (Color online) End facet of WG2 as seen with different characterization methods: (a) Transmission white light micrograph (b) Guided mode at $1550 \mathrm{~nm}$. (c) Spectral shift in $\mathrm{cm}^{-1}$ of the $553 \mathrm{~nm}$ Erbium emission line as obtained under $488 \mathrm{~nm}$ excitation. (d) Spectral shift in $\mathrm{cm}^{-1}$ of the $976 \mathrm{~nm}$ emission line as obtained under $488 \mathrm{~nm}$ excitation. (e) Normalized $976 \mathrm{~nm}$ Ytterbium emission intensity as obtained under $488 \mathrm{~nm}$ excitation. (f) Normalized $657 \mathrm{~nm}$ upconversion emission intensity of the Erbium ions under $980 \mathrm{~nm}$ excitation. 
case) over the same region. Using the same irradiation parameters it is possible to produce a refractive index increase ${ }^{12}$ (WG2). Figure 3(a) shows a white light transmission micrograph of the end facet of WG2 together with its guided mode at $1550 \mathrm{~nm}$ [Fig. 3(b)]. As for WG1, the Erbium emission shift image at $553 \mathrm{~nm}$ [Fig. 3(c)] reveals an outer region displaying a blueshift. Interestingly, the blue shifted emission is limited to the outer irradiated region, indicating matrix expansion and lower density and refractive index only there. Figure 3(d) shows the spectral shift image at $976 \mathrm{~nm}$ under excitation at $488 \mathrm{~nm}$, featuring a red-shift of the $\mathrm{Yb}$ ion emission in the central region, which was not present in WG1. As in the case of Erbium ions, we are not aware of any previous study concerning the spectral shift of this line under the application of compressive or tensile stress in glasses. Nevertheless, and in a first order approximation, we attribute this red-shift in the Ytterbium emission to an increase in the averaged crystal field and hence, to a local compression of the network surrounding the Ytterbium ions, indicative of a local increase of the refractive index.

By comparing Figs. 3(c) and 3(d), complementary information can be obtained. It shows that in our conditions, the Er ion peak emission shift at $553 \mathrm{~nm}$ is more affected by matrix expansion, while the emission of the $\mathrm{Yb}$ ion at $976 \mathrm{~nm}$ is more sensitive to the compression of the matrix. In this way, it can be concluded that the outer region features a decrease in density (and thus a refractive index decrease), and that the inner region experiences compression, and thus, a refractive index increase.

This complex distribution of the material's local density also affects PL and the transfer properties of the Er and $\mathrm{Yb}$ ions as shown in Figs. 3(e)-3(f). As for WG1, the density reduction in the outer region in WG2 implies a decrease in the $\mathrm{Er} \rightarrow \mathrm{Yb}$ energy transfer, and thus a drop in $\mathrm{Yb}$ peak emission at $976 \mathrm{~nm}$ upon excitation at $488 \mathrm{~nm}$ as shown in Fig. 3(e). In contrast, in the central densified region, the Er $\rightarrow$ Yb local energy transfer gets improved which manifests as an increase in the $\mathrm{Yb}$ emission at $976 \mathrm{~nm}$.

The central densification should also increase $\mathrm{Yb} \rightarrow \mathrm{Er}$ energy transfer with respect to that observed in WG1. By comparing the Er UC image of WG2 [Fig. 3(f)] to the corresponding one of WG1 [Fig. 2(f)], a relative increase in the central region can indeed be appreciated. At this point it has to be emphasized that the fluorescence red- and blueshifts are signatures of the modification of the crystal field, and thus, of the local density of the material. We could expect some contribution to the refractive index modification induced upon irradiation due to changes in the local polarizability of the material (i.e., bond breaking). This would usually be accompanied by a fluorescence quenching and an overall reduction of the $\mathrm{Er}^{3+}$ and $\mathrm{Yb}^{3+}$ fluorescence, which is not experimentally observed. Bond breaking effects, even if present, look as a second order effect for explaining the observed refractive index changes.

Gathering all results obtained from Fig. 3, we can conclude that the origin of guiding in WG2 stems from a central densification which generates an increase in the refractive index together with an outer annular decrease in density and refractive index. Additionally, this density depressed region has the effect of increasing the refractive index contrast among the central and outer region. It is worth noting that the mechanism proposed by Little et al. ${ }^{8}$ for the refractive index increase in Kigre Inc. QX-glass under their processing conditions is different from ours, even though in both cases processing has been done in the low repetition rate regime. When comparing our results to those in Ref. 8, we cannot conclude whether the structural modification of the glass matrix leading to a refractive index increase is originated by the different matrix composition or by the different processing conditions. In this context it is important to recall that in our case the positive refractive index change has been achieved upon multiple re-scans. The accumulative effects coming out of this strategy lead to a different modification mechanism, apparently closer to that found in the high repetition rate regime as reported by Fletcher et al. ${ }^{7}$ in MM2 glass, where thermal accumulation effects were present. The importance of different writing conditions is clearly shown in a study by Ams et al., ${ }^{19}$ who investigate the same material (QX) with a low-repetition and a high repetition rate system. For both repetition rate systems they identify irradiation intensity windows and focusing conditions to achieve positive and negative refractive index changes.

\section{SUMMARY}

In the present work we have demonstrated the potential of confocal fluorescence imaging, a technique used for crystals, to understand the origin of local modifications in fslaser processed doped phosphate glasses. In particular, this work has been focused on a commercial Er-Yb codoped phosphate glass of interest for photonic devices. We have revealed clear differences in the PL and UC emission of the irradiated regions compared to those of the nonirradiated regions. We have also been able to establish consistent relations between PL intensity, PL spectral shift and UC intensity behaviors in terms of $\mathrm{Er} \rightarrow \mathrm{Yb}$ and $\mathrm{Yb} \rightarrow$ Er energy transfer mechanisms. Finally, we have not only identified the regions where the refractive index has been modified upon fs-laser irradiation as a consequence of the material's local change in density, but we have also identified the local sign of the change in each case.

\section{ACKNOWLEDGMENTS}

This work was partially supported by the Spanish Ministry of Science and Innovation (Grant Nos.TEC2008-01183 and MAT 2010-16161) and the Comunidad Autónoma de Madrid (PHAMA P2009/MAT1756). A.R. acknowledges a I3P-CSIC postdoctoral contract (co-funded by the European Social Fund). A.F. acknowledges a Grant under Project TEC 2006-04538.

${ }^{1}$ K. M. Davis, K. Miura, N. Sugimoto and K. Hirao, Opt. Lett. 21, 1729 (1996). ${ }^{2}$ S. Taccheo, G. Della Valle, R. Osellame, G. Cerullo, N. Chiodo, P. Laporta, O. Svelto, A. Killi, U. Morgner, M. Lederer and D. Kopf, Opt. Lett. 29, 2626 (2004).

${ }^{3}$ A. M. Streltsov and N. F. Borrelli, Opt. Lett. 26, 42 (2001).

${ }^{4}$ C. Hnatovsky, R. S. Taylor, E. Simova, P. P. Rajeev, D. M. Rayner, V. R. Bhardwaj and P. B. Corkum, Appl. Phys. A84, 47 (2006). 
${ }^{5}$ R. Martinez Vazquez, R. Osellame, D. Nolli, C. Dongre, H. van den Vlekkert, R. Ramponi, M. Pollnau and G. Cerullo, Lab Chip 9, 91 (2009).

${ }^{6}$ A. Saliminia, N. T. Nguyen, S. L. Chin and R. Vallée, J. Appl. Phys. 99, 093104 (2006).

${ }^{7}$ L. B. Fletcher, J. J. Witcher, W. B. Reichman, A Arai, J. Bovatsek and D. M. Krol, J. Appl. Phys. 106, 083107 (2009).

${ }^{8}$ D. J. Little, M. Ams, P. Dekker, G. D. Marshall and M. J. Withford, J. Appl. Phys. 108, 033110 (2010).

${ }^{9}$ A. Ródenas, G. A. Torchia, G. Lifante, E. Cantelar, J. Lamela, E. Jaque, L. Roso and D. Jaque, Appl. Phys. B95, 85 (2009).

${ }^{10}$ Y. Cheng, K. Sugioka, K. Midorikawa, M. Masuda, K. Toyoda, M. Kawachi and K. Shihoyama, Opt. Lett. 28, 55 (2003).

${ }^{11}$ W. Gawelda, D. Puerto, J. Siegel, A. Ferrer, A. Ruiz De La Cruz, H. Fernández, and J. Solis, Appl. Phys. Lett. 93 (2008).
${ }^{12}$ A. Ferrer, A. Ruiz De La Cruz, D. Puerto, W. Gawelda, J. A. Vallés, M. A. Rebolledo, V. Berdejo, J. Siegel and J. Solis, J. Opt. Soc. Am. B 27, 1688 (2010).

${ }^{13}$ M. Ams, G. D. Marshall and M. J. Withford, Opt. Express 14, 13158 (2006).

${ }^{14}$ D. Low, H. Xie, Z. Xiong and G. Lim, Appl. Phys. A 81, 1633 (2005).

${ }^{15}$ K. Hirao and K. Miura, J. Non-Cryst. Solids 239, 91 (1998).

${ }^{16}$ B. Henderson and G. F. Imbusch, Optical Spectroscopy of Inorganic Solids (Oxford University Press, New York,1989).

${ }^{17}$ G. Liu and B. Jacquier, Spectroscopic Properties of Rare Earths in Optical Materials, (Springer, New York, 2005).

${ }^{18}$ J. A. Vallés, M. Á. Rebolledo, V. Berdejo, A. Ferrer, A. Ruiz de la Cruz and J. Solís, Opt. Mater. 33, 231 (2010).

${ }^{19}$ M. Ams, G. D. Marshall, P. Dekker, M. Dubov, V. K. Mezentsev, I. Bennion and M. J. Withford, IEEE J. Sel. Top. Quantum Electron. 14, 1370 (2008). 